This item was submitted to Loughborough's Research Repository by the author.

Items in Figshare are protected by copyright, with all rights reserved, unless otherwise indicated.

\title{
Evaporation of droplets of surfactant solutions
}

PLEASE CITE THE PUBLISHED VERSION

http://dx.doi.org/10.1021/la401578v

\section{PUBLISHER}

() American Chemical Society (ACS)

\section{VERSION}

AM (Accepted Manuscript)

\section{PUBLISHER STATEMENT}

This work is made available according to the conditions of the Creative Commons Attribution-NonCommercialNoDerivatives 4.0 International (CC BY-NC-ND 4.0) licence. Full details of this licence are available at: https://creativecommons.org/licenses/by-nc-nd/4.0/

\section{LICENCE}

CC BY-NC-ND 4.0

\section{REPOSITORY RECORD}

Semenov, Sergey, Anna Trybala, Hezekiah Agogo, Nina Kovalchuk, Francisco Ortega, Ramon Rubio, Victor Starov, and M.G. Velarde. 2017. "Evaporation of Droplets of Surfactant Solutions". figshare.

https://hdl.handle.net/2134/25792. 


\section{Evaporation of droplets of surfactant solutions}

Sergey Semenov ${ }^{\mathrm{a}}$, Anna Trybala ${ }^{\mathrm{a}}$, Hezekiah Agogo ${ }^{\mathrm{b}, \mathrm{c}}$, Nina Kovalchuk ${ }^{\mathrm{a}, \mathrm{d}}$, Francisco Ortega ${ }^{\mathrm{b}}$, Ramón G. Rubio ${ }^{\mathrm{b}, \mathrm{c}^{*}}$, Víctor M. Starov ${ }^{\mathrm{a}^{*}}$, Manuel G. Velarde ${ }^{\mathrm{c}}$

${ }^{a}$ Department of Chemical Engineering, Loughborough University, Loughborough, UK. bDepartamento de Química Física I, Facultad de Química, Universidad Complutense, 28040 Madrid, Spain.

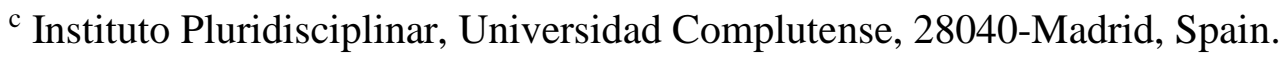

${ }^{\mathrm{d}}$ Institute of Biocolloid Chemistry, Kiev, Ukraine.

*To whom correspondence should be addressed: rgrubio@quim.ucm.es; v.m.starov@lboro.ac.uk 


\section{Abstract}

The simultaneous spreading and evaporation of droplets of aqueous trisiloxane (superspreader) solutions onto a hydrophobic substrate has been studied both experimentally, using a video-microscopy technique, and theoretically. The experiments have been carried out over a wide range of surfactant concentration, temperature and relative humidity. Similar to pure liquids, four different stages have been observed: the initial one corresponds to spreading till the contact angle, $\theta$, reaches the value of the static advancing contact angle, $\theta_{\text {ad. }}$. Duration of this stage is rather short and the evaporation during this stage can be neglected. The evaporation is essential during next three stages. The next stage after the spreading, which is referred to below as the first stage, takes place at constant perimeter and ends when $\theta$ reaches the static receding contact angle, $\theta_{\mathrm{r}}$. During the next, second stage, the perimeter decreases at constant contact angle $\theta=\theta_{\mathrm{r}}$ for surfactant concentration above critical wetting concentration (CWC). The static receding contact angle decreases during the second stage for concentrations below CWC because the concentration increases due to the evaporation. During the final stage both the perimeter and the contact angle decrease till the drop disappears. Below we consider only the longest stages one and two.

The developed theory predicts universal curves for the contact angle dependency on time during the first stage, and for the droplet perimeter on time during the second stage. A very good agreement between theory and experimental data has been found for the first stage of evaporation, and for the second stage for concentrations above CWC, however, some deviations were found for concentrations below CWC. 


\section{Introduction}

Evaporation of liquid droplets in a gas volume has implications in different areas: spray drying and production of fine powders [1-3], spray cooling [4-6], fuel preparation [710], air humidifying [11], heat exchangers [12], drying in evaporation chambers of air conditioning systems [11, 12], fire extinguishing [13, 14], fuel spray auto ignition (Diesel) [15], solid surface templates from evaporation of nanofluid drops (coffee-ring effect) [16], spraying of pesticides [1-3], painting, coating and inkjet printing [17], printed MEMS devices, micro lens manufacturing, spotting of DNA microarray data [3, 18-19]. Because of such wide range of industrial applications this phenomenon has been under investigation for many years, both for pure fluids and for complex fluids. The studies encompass different conditions: constant pressure and temperature, elevated pressure, fast compression, still gas atmosphere and turbulent reacting flows, strongly and weakly pinning substrates [1, 2]. The experimental, theoretical and computer simulation studies carried out so far [1-3, 18-27] have taken into account different physical processes: heat transfer inside droplets, mass diffusion in bi- and multicomponent fluids, droplet interactions in sprays, turbulence, radiation absorption, thermal conductivity of the solid substrate, Marangoni convection inside the droplets. However a comprehensive knowledge of the phenomenon is still lacking, especially for complex fluids (surfactant solutions, suspensions, etc.).

Based on [1, 27-28] one can summarize results for the evaporation of a drop of a pure fluid onto a smooth surface under partial wetting conditions:

a) If droplet is big enough [29, 30] then the evaporation is limited by vapour diffusion into surrounding air and the evaporation rate is proportional to the radius of the droplet base, L. 
b) The spreading and evaporation process is composed of four stages (Figure 1): Spreading stage. During this short stage immediately after a deposition both the contact angle and radius changes simultaneously reaching in the end values $\theta_{\mathrm{ad}}$ and $\mathrm{L}_{0}$, correspondingly. These values are used as initial values for the following first stage. As discussed in detail by Svitova et al. [31] and Ivanova et al. [32], during the spreading process (initial stage) it is possible to use a power-law dependency of the contact angle on time. In our experiments the characteristic time scale of the initial stage of spreading was found to be in the range of $50 \mathrm{~s}$. This value is similar to those found in Ref. [32] for aqueous trisiloxane solutions. This stage is short enough, less than $100 \mathrm{sec}$, and the volume change is less than 5\% [32]. Hence, it is possible to neglect evaporation during this stage. It is the reason why this stage is not considered below. Stage I. The contact angle decreases from $\theta_{a d}$ down to static receding contact angle value, $\theta_{r}$, at constant $L$. Stage II. Contact angle remains constant and equal its receding value, $\theta_{r}$, while the radius of the droplet base, $L$, decreases. Stage III. Both the contact angle, $\theta$, and the radius of the droplet base, $L$ decrease until the drop completely disappears. This stage is also relatively shorter as compared with the stages one and two. Probably surface forces (disjoining/conjoining pressure) become important on this stage [33, 34]. Some consideration of the third stage is undertaking below.

Introducing a dimensionless contact line radius and dimensionless times for the first and second stages of evaporation allowed obtaining universal laws describing the experimental data for water droplets onto various substrates in the presence of contact angle hysteresis [1, 27-28]. Based on these results a model was proposed that was capable of explaining quantitatively the first and second stages of the evaporation of pure fluids. 


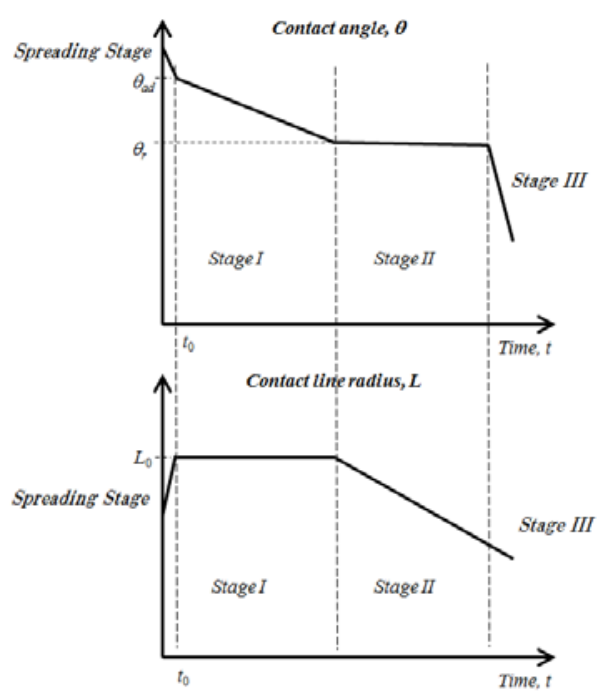

Figure 1. Four stages of spreading/evaporation. Below only two longest stages I and II are under consideration.

Mixtures of fluids and surfactant solutions have also been studied in the last few years. Sefiane et al. [35], Soboleva and Summ [36], Gutiérrez et al. [37], Gokhale et al. [38] and Alexandridis et al. [39] have carried out experiments on kinetics of evaporation of droplets of surfactant solutions. Their results have led to a conclusion that the surfactants play an important role in the spreading and evaporation of droplets of surfactant solutions: the presence of surfactants favoured higher values of $L$ on hydrophobic substrates (due to the decrease of the contact angle), and therefore higher evaporation rates [35].

In spite of all the above mentioned works, a number of problems still remain to be solved: a) to build a theory for drops of multi-component fluids (including surfactant solutions); b) to build a hydrodynamic model capable of describing the four stages of the simultaneous spreading and evaporation process; c) to match the description of spreading/evaporation of the drop in the bulk with the thin layer behind the apparent three-phase contact line where surface forces are important [33, 34]. Such matching 
must take into consideration the DLVO forces acting at a mesoscopic scale near the contact line; d) to describe the evaporation process of complex fluids: polymer and protein solutions and nanoparticle suspensions; e) to build a theory capable of describing the evaporation of drops onto patterned surfaces.

In the case of surfactant solutions the situation is even more complex because, in addition to the previous problems, it is necessary to take into account that below critical aggregation concentration (CAC) the adsorption at both the vapour-liquid and the liquid-solid interfaces (and, hence, the corresponding interfacial tensions) are concentration dependent, and therefore can change during the evaporation process. This will introduce a contribution to the time dependence of $\theta$ that was not considered earlier by the suggested theory for pure liquids.

The aim of this work is to perform a detailed experimental study of the time dependence of the contact angle, the volume and the radius of the droplet base of aqueous trisiloxane surfactant solution onto a hydrophobic TEFLON-AF substrate. This substrate has been chosen because a slow spreading on this highly hydrophobic substrate allows extracting more information as compared to moderately hydrophobic solid substrates [26]. We used drops of an aqueous solution of a superspreader surfactant (SILWET L77) over a wide concentration range. Below only the experimental data obtained for the first and second stages are compared with the theoretical and computer simulation results.

\section{Theory}

Model of diffusion limited evaporation in the case of contact angle hysteresis developed in [1, 27-28] is discussed below. 
The geometry of evaporation of a spherical cap droplet could be found in Supporting Information (Figure S1). We assume that during the first (constant radius of the droplet base) and second (constant contact angle) stages of evaporation the drop remains spherical. In this case its volume, $\mathrm{V}$, is given by the following relation:

$$
V=L^{3} f(\theta) \quad f(\theta)=\frac{\pi}{3} \frac{(1-\cos \theta)^{2}(2+\cos \theta)}{\sin ^{3} \theta}
$$

where $L$ is the radius of the droplet base, $\theta$ is the contact angle. According to [1, 27-28] the evaporation rate can be calculated in the following way:

$$
\frac{\mathrm{dV}}{\mathrm{dt}}=-\beta \mathrm{F}(\theta) \mathrm{L},
$$

where

$$
\begin{aligned}
& F(\theta)= \begin{cases}\left(0.6366 \cdot \theta+0.09591 \cdot \theta^{2}-0.06144 \cdot \theta^{3}\right) / \sin \theta, & \theta<\pi / 18 \\
\left(0.00008957+0.6333 \cdot \theta+0.116 \cdot \theta^{2}-0.08878 \cdot \theta^{3}+0.01033 \cdot \theta^{4}\right) / \sin \theta, & \theta \geq \pi / 18\end{cases} \\
& \beta=2 \pi \frac{\mathrm{DM}}{\rho}\left[\mathrm{c}_{\text {sat }}\left(\mathrm{T}_{\mathrm{av}}\right)-\mathrm{c}_{\infty}\right],
\end{aligned}
$$

where $T_{a v}=\int T_{\text {surf }} d S$ is the average temperature of the liquid-air droplet surface, $S$, $\mathrm{T}_{\text {surf }}$ is the temperature of the liquid/vapour interface, $D$ is the diffusion coefficient of the liquid vapour in air, $\rho$ is the liquid density, $M$ its molecular weight, $C_{\text {sat }}$ is the water vapour concentration on the droplet surface equal to the concentration of the saturated vapour, $c_{\infty}=H \cdot c_{\text {sat }}\left(T_{\infty}\right)$ is the water vapour concentration in the air far from the droplet surface, and $H$ is the relative humidity of the ambient air. It was suggested in [1, 27-28] that for pure fluids the average temperature of the droplet-air interface, $T_{a v}$, can be taken as a constant during the evaporation process. Thus at constant values of the ambient temperature, $T_{\infty}$, and the relative humidity, $H$, coefficient $\beta$ remains constant during the 
evaporation process. Eq.(2) indicates that the evaporation rate is proportional to the perimeter of the drop. This has been proved theoretically earlier [1, 27-28].

Note, the function (3) was calculated in [40] for isothermal conditions. However, it was shown earlier [3] that the temperature inside a droplet in the course of evaporation remains constant. An average surface temperature, $T_{a v}$, [1, 27-28] has been introduced and based on [3] it was assumed that $T_{a v}$ also remains constant in the course of evaporation.

\section{Calculation of parameter $\beta$}

The previous consideration shows that $\beta$ is the only parameter in the above theory (see Equations (2) and (4)). Below this parameter is calculated according to method suggested in [28].

In the experimental study performed an evaporating droplet has been situated on the solid substrate composed of three layers. The lower layer is glass in contact with air, the intermediate one is a silicon wafer and the upper layer in contact with droplet is a Teflon film. The computational scheme was adopted accordingly. The schematic presentation of the solid support used for measurements of the evaporation of aqueous surfactant solutions can be found in Supporting Information (Figure S2).

The following geometrical and physical parameters were selected for calculations: geometrical parameters: radius of the droplet base, $\mathrm{L}=1 \mathrm{~mm}$, contact angle, $\theta=95^{0}$, thickness of Teflon layer $1 \mu \mathrm{m}$, thickness of the silicon wafer is $600 \mu \mathrm{m}$; 
physical parameters of water: density $1000 \mathrm{~kg} / \mathrm{m}^{3}$, dynamic viscosity $10^{-3} \mathrm{~Pa} \cdot \mathrm{s}$, airliquid interfacial tension, $\gamma=0.030 \mathrm{~N} / \mathrm{m}, \mathrm{d} \gamma / \mathrm{dT}=-1.7 \mathrm{e}-4 \mathrm{~N} /(\mathrm{m} \cdot \mathrm{K})$, thermal conductivity $0.58 \mathrm{~W} /(\mathrm{m} \cdot \mathrm{K})$, specific heat capacity $4200 \mathrm{~J} /(\mathrm{kg} \cdot \mathrm{K})$, latent heat of vaporization 44320 $\mathrm{J} / \mathrm{mol}$, molar mass $0.018 \mathrm{~kg} / \mathrm{mol}$;

physical parameters of air and vapour; tabulated values of saturated vapour pressure at corresponding temperatures, diffusion coefficient of vapour in air $2.4 \mathrm{e}-5 \mathrm{~m}^{2} / \mathrm{s}$, Air density $1.184 \mathrm{~kg} / \mathrm{m}^{3}$, dynamic viscosity 2e-5 Pa.s, thermal conductivity 0.0243 $\mathrm{W} /(\mathrm{m} \cdot \mathrm{K})$, specific heat capacity $1005 \mathrm{~J} /(\mathrm{kg} \cdot \mathrm{K})$;

physical parameters of glass: density $2400 \mathrm{~kg} / \mathrm{m}^{3}$, thermal conductivity $1.2 \mathrm{~W} /(\mathrm{m} \cdot \mathrm{K})$, specific heat capacity700 J/(kg·K);

physical parameters of silicon wafer: density $2330 \mathrm{~kg} / \mathrm{m}^{3}$, thermal conductivity 149 $\mathrm{W} /(\mathrm{m} \cdot \mathrm{K})$, specific heat capacity $700 \mathrm{~J} /(\mathrm{kg} \cdot \mathrm{K})$;

physical parameters of Teflon: density $2200 \mathrm{~kg} / \mathrm{m}^{3}$, thermal conductivity 0.25 $\mathrm{W} /(\mathrm{m} \cdot \mathrm{K})$, specific heat capacity $1300 \mathrm{~J} /(\mathrm{kg} \cdot \mathrm{K})$.

Parameter $\beta$ was extracted from the results of computer simulations as $\beta=\frac{J}{\rho_{l} F(\theta) L}$, where $J$ is the total mass flux of droplet evaporation, $\rho_{l}$ is water density, $L$ is the radius of the droplet base, $F(\theta)$ is the function of contact angle determined by Picknett and Bexon [40] according to Eq. (3). Numerical modelling described in [28] was used to predict the dependence of $\beta$ on the ambient temperature, $T_{\infty}$ and humidity $H$. Note that this model does not take into account the dependence of diffusion coefficient, $D$, on temperature. 
Computer simulations have shown that the parameter $\beta$ decreases with increasing relative humidity, $H$, at constant ambient temperature, $T_{\infty}$, and increases with temperature at constant humidity (see Figures S3 and S4 in supporting information). The experimental results confirm the trends shown by the computer calculations.

However, it has to be noted that according to Equation (2) the evaporation rate depends not solely on $\beta$ but also on the contact angle $\theta$ (through $F(\theta)$ ) and the radius of the droplet base $L$. The most important one (see below) is the dependency of the static receding contact angle on surfactant concentration of the solutions in the range $0<C \leq \mathrm{C}_{\mathrm{cr}}$ (where $\mathrm{C}_{\mathrm{cr}}=\mathrm{CWC}$ for SILWET L77 or $\mathrm{C}_{\mathrm{cr}}=\mathrm{CMC}$ for SDS). The concentration inside the droplet varies over time and so does the static receding contact angle. However, the static receding contact angle does not vary any more if surfactant concentration is above CWC/CMC.

Why does the time dependence of contact angle not influence the time dependency of volume on time?

In all our experiments (see below and also [20]) a linear dependence $V^{2 / 3}(t)=V_{0}^{2 / 3}-$ const $\cdot t$ was found, where $\mathrm{V}(\mathrm{t})$ is the dependence of the volume of evaporating droplet on time. The latter conclusion looks like it is in contradiction with Equation (2), which describes the dependence of the evaporation rate on the contact angle. Below we show that in spite of that the linear dependence is still approximately valid.

From Equation (1) we can express the radius of the droplet base, L, via the droplet volume, $\mathrm{V}$, and the contact angle, $\theta$. Substitution into Equation (2) results in: 
$\frac{d V}{d t}=-\beta F(\theta) \frac{V^{1 / 3}}{f^{1 / 3}(\theta)}$

Let us introduce the following function of contact angle:

$$
\frac{F(\theta)}{f^{1 / 3}(\theta)}=\Lambda(\theta)
$$

In our measurements all receding and advancing contact angle were inside the following range from $43^{\circ}$ to $115^{\circ}$. Calculations according to Eq. (6) shows that in this range of contact angles $\Lambda(\theta)$ varies from 0.79 to 0.86 . This means that $\Lambda(\theta)$ can be considered approximately as a constant, $\Lambda$, with a reasonable degree of approximation when the contact angle ranges between $\theta_{\mathrm{ad}}$ and $\theta_{\mathrm{r}}$. The constant $\Lambda$ is independent of the surfactant concentration.

Note, that $\Lambda(\theta)$ changes only slightly during the first stage of evaporation. During the second stage of evaporation the contact angle remains constant and so does $\Lambda\left(\theta_{\mathrm{r}}\right)$.

The latter means that we can rewrite Equation (5) as:

$\frac{d V}{d t}=-\alpha V^{1 / 3}$, where $\alpha=\beta \Lambda$

Integration of the latter equation results in: $V^{2 / 3}(t)=-\frac{2 \alpha}{3} t+V_{0}^{2 / 3}$. Introducing in this equation dimensionless time $\hat{\tau}=\frac{\beta t}{V_{0}^{2 / 3}}$ we can rewrite it as

$$
\left(\frac{V(t)}{V_{0}}\right)^{2 / 3}=1-\frac{2 \Lambda}{3} \hat{\tau}
$$

All experimental dependences of volume on time obtained for all concentrations studied agree with the linear dependence given by Eq. (8) (see Figure 2). Note, that 
concentrations in Figure 2 are normalized by CAC (for SILWET L77 CAC=0.1 g/l [41])

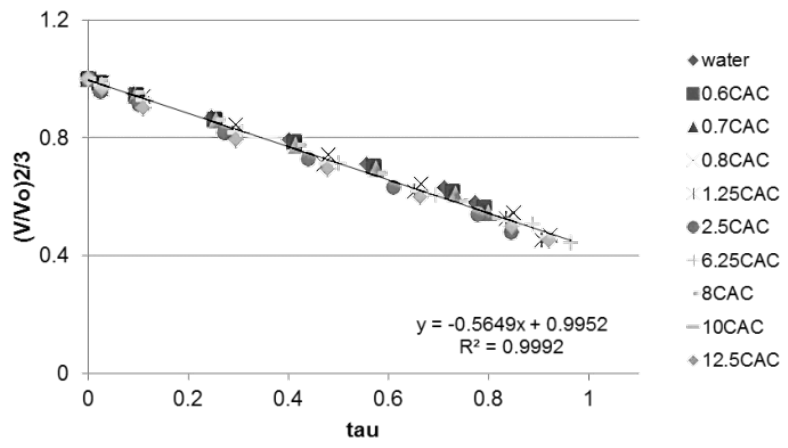

Figure 2. An experimental dependency for different SILWET L77 concentration at $24^{0}$ $\mathrm{C}$ and $50 \%$ humidity. The value of $\beta$ is taken from computer simulations (Figures S3 and S4 in Supporting Information).

Figure 2 shows an excellent linear fit, which gives $\frac{2}{3} \Lambda=0.5649$ (according to Equation (8)) and, hence, $\Lambda=0.84735$, that is, inside the mentioned above range (from 0.79 to 0.86 ). All other experimental dependences of volume on time follow the linear trend predicted by Equation (8).

Dimensionless variables and universal dependences for two stages of evaporation.

During the first stage of spreading/evaporation process radius of the droplet base, L, remains constant and equal to its value, $\mathrm{L}_{0}$, in the end of the spreading stage. Then Eq.(2) can be rewritten as

$$
\mathrm{L}_{0}^{2} \cdot \mathrm{f}^{\prime}(\theta) \frac{\mathrm{d} \theta}{\mathrm{dt}}=-\beta \cdot \mathrm{F}(\theta)
$$


from which time dependence of the contact angle can be easily obtained after numerical integration. The latter theory predicts a universal behaviour for this evaporation stage when the variables are expressed in terms of a reduced time, $\tilde{\tau}$, defined as:

$$
\tilde{\tau}=\tau+\int_{\theta_{a d}}^{\pi / 2} f^{\prime}(\theta) / F(\theta) d \theta, \quad \tau=t / t_{c h}, \quad t_{c h}=L_{0}^{2} / \beta
$$

The upper limit of integration, $\pi / 2$, was arbitrary chosen.

For the second stage of spreading/evaporation process the initial value of contact angle is $\theta_{\mathrm{r}}$. During the second evaporation stage the contact angle $\theta$ remains constant and equals to it static receding value $\theta_{r}$ in the case of pure liquids. The theory [28] also predicts that during the second stage of evaporation $l(\theta)=\left[1-\frac{2 F\left(\theta_{r}\right)}{3 f\left(\theta_{r}\right)}\left(\tau-\tau_{r}\right)\right]^{1 / 2}$, where $l=\mathrm{L} / \mathrm{L}_{0}$ is a reduced radius of the droplet base, and reduced time $\tau_{r}$ corresponds to the moment when receding starts. The latter dependency represents a universal behaviour for the time dependence of the reduced radius of the droplet base, $l$, on reduced time during the second stage of evaporation. In terms of a new reduced time $\bar{\tau}=\frac{2 F\left(\theta_{r}\right)}{3 f\left(\theta_{r}\right)}\left(\tau-\tau_{r}\right)$ it takes the following form:

$$
l(\bar{\tau})=(1-\bar{\tau})^{1 / 2}
$$

In deriving the universal curve for the second stage, $\widetilde{\tau}=0$ was arbitrarily chosen as $\theta=$ $\pi / 2$. Thus, negative values of the reduced time, $\tilde{\tau}<0$, at the second stage correspond to contact angles $\theta>\pi / 2$. If the surface is hydrophobic $(\theta>\pi / 2)$ then it corresponds to a negative time on the universal dependence. The theoretical predictions were found to agree well with the available data for water droplets onto different solid substrates as 
shown in Figure 3 [28]. Figure 3 shows that agreement with the theory for the both stages is very good.

Important to note that to plot universal dependences presented in Figure 3 we used experimental values of both advancing and receding contact angles. Those values cannot be predicted in the framework of the above theory.
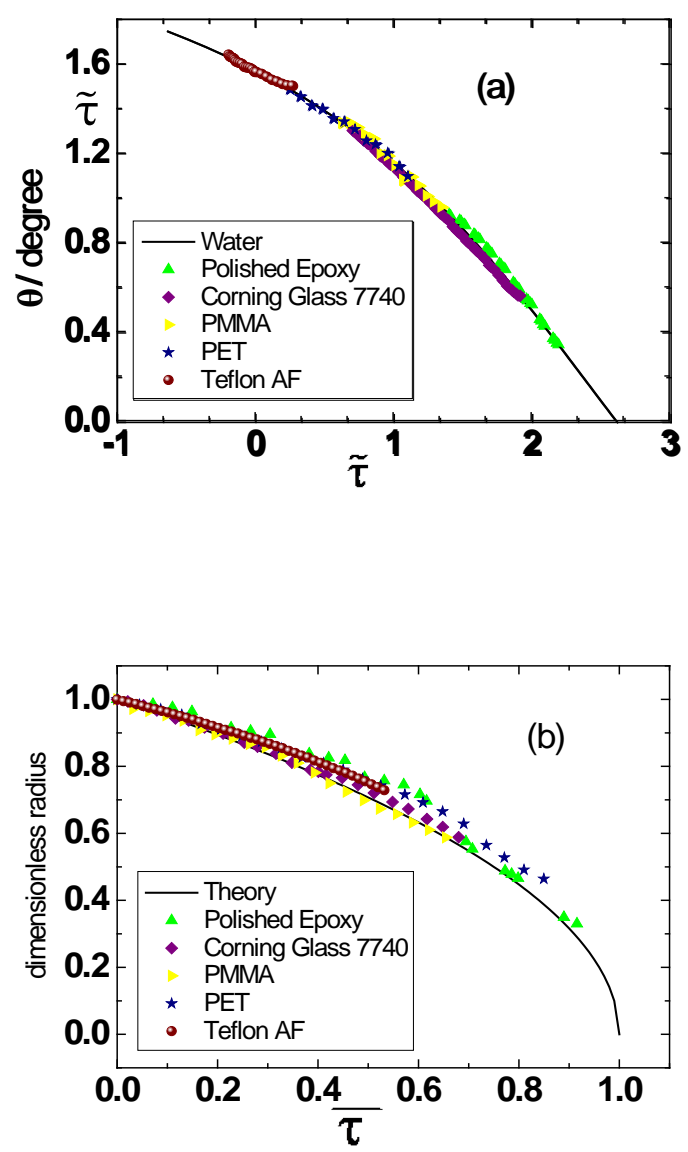

Figure 3. Comparison of experimental results taken from various literature sources for evaporation of water droplets onto hydrophobic substrates with the universal curves predicted by the theory for the first and second stages of the evaporation process shown in (a) and (b) respectively. Redrawn from [28].

Experimental. 
SILWET L77 was purchased from Sigma-Aldrich (Germany) and used as received. Poly(4, 5-difluoro-2, 2-bis(trifluorimethyl)-1， 3-dioxole-co-tetrafluoroethylene), hereinafter TEFLON-AF, was purchased from Sigma-Aldrich (Germany) as powder, the Flourinet F-77 solvent was bought from 3M (USA), and the silicon wafers were obtained from Siltronix (France). Ultrapure deionized water (Younglin Ultra 370 Series, Korea) with a resistivity higher than $18 \mathrm{M} \Omega$ and TOC lower than 4 ppm was used for preparing all the surfactant solutions.

All surfactant solutions were prepared by weight using a balance precise to $\pm 0.01 \mathrm{mg}$. A $\mathrm{pH} 7.0$ buffer was used as a solvent to prevent hydrolysis of the SILWET L77. The solutions were used immediately after preparation. It was checked that the buffer did not changed the surface tension of water and that fresh SILWET L77 solutions with and without buffer have the identical surface tension. The silicon wafers were cleaned using piranha solution for 20 min (caution piranha solution is highly oxidizing!). The solid substrates were prepared as follows: the TEFLON-AF powder was suspended in the Flourinet F77 and spin-coated onto the silicon wafers. The average roughness of the $20 \mu \mathrm{m} \times 20 \mu \mathrm{m}$ surface was $\approx 1.0 \mathrm{~nm}$ as measured by AFM (tapping mode). The static advancing contact angle of pure water on this substrate is $118 \pm 2^{\circ}$, which agrees with [32]. Drops of $4 \mathrm{~mm}^{3}$ were deposited onto the substrate for measurements. Five independent measurements were performed for each experimental point reported in this work and the average was used.

The experimental technique used was similar to the one used earlier by Ivanova et al.[26, 32] with some modifications that allowed us to monitor continuously the temperature and the relative humidity inside the experimental cell. A diagram of the 
experimental device is shown in Fig. S5 of Supporting information. The cameras were calibrated using a micro-ruler with a precision of $\pm 0.5 \mu \mathrm{m}$.

Sessile drops were deposited onto the substrate inside a chamber attached to a thermostat, and their shape and size were captured by the CCD camera (side view) at 30 fps. The initial drop volumes used were about $4 \mathrm{~mm}^{3}$ in order to ensure that gravity effects can be neglected and the drop always had a spherical cap shape. The images captured were analyzed using the drop tracking and evaluation analysis software (Micropore Technologies, UK) that allowed monitoring the time evolution of diameter the drop base, height, radius of the curvature, and contact angle. The precision of the contact angle measurements was $\pm 2^{\circ}$ under dynamic conditions, i.e. spreading and evaporation; those of height and diameter were $\pm 1 \mu \mathrm{m}$ and that of the temperature was \pm

$0.5^{0} \mathrm{C}$. The relative humidity, $\mathrm{H}$, was maintained constant by placing saturated salt solutions inside the measuring chamber and it was measured with a precision of $\pm 2 \%$.

\section{Experimental results and Discussion}

Figure 1 shows a qualitative behaviour of contact angle, $\theta$, and the radius of the droplet base, $L$, for pure aqueous droplets. According to our experimental results similar behaviour during studied here stages I and II was observed also for aqueous SILWETT L77 solution at concentration above CWC (CWC $=0.40 \mathrm{mmol} / \mathrm{l}=0.25 \mathrm{~g} / \mathrm{l}$ being the critical wetting concentration of the SILWET L77, [32]).

The experimental dependences of the droplet volume show that $\mathrm{V}^{2 / 3}$ decreases linearly with time during all first three stages of the process (see Figure 2), as in the case of SDS solutions [20]. It was shown above that the latter linear dependency is not in a contradiction with dependency of the evaporation rate on the contact angle according to Equation (2). 
The time dependence of the radius of the droplet base for all solutions studied, independently of concentration is similar to that presented schematically in Fig. 1 with $\mathrm{L}_{0}$ increasing as the surfactant concentration increases.

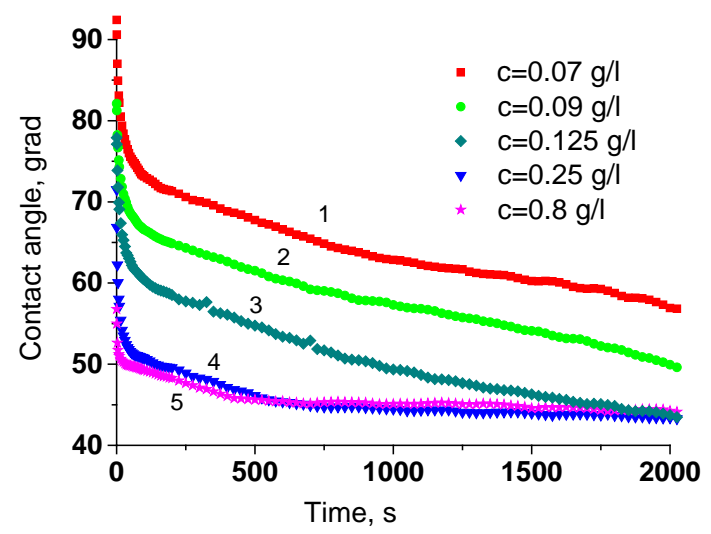

Figure 4. Time dependence of the contact angle for different concentrations of SILWETT L77 surfactant at $24^{\circ} \mathrm{C}$ and 50\% relative humidity: $1-\mathrm{c}=0.07 \mathrm{~g} / \mathrm{l}, 2-\mathrm{c}=0.09$ $\mathrm{g} / \mathrm{l}, 3-\mathrm{c}=0.125 \mathrm{~g} / \mathrm{l}, 4-\mathrm{c}=0.25 \mathrm{~g} / \mathrm{l}(\mathrm{CWC}), 5-\mathrm{c}=0.8 \mathrm{~g} / \mathrm{l}$. The final third stage is not shown.

Figure 4 shows time dependences of contact angle, $\theta$, for the SILWETT L77 solutions over the investigated concentration range (not all concentrations are shown for the sake of clarity). As expected, the increase of surfactant concentration reduces both the initial contact angle (at the beginning of the spreading stage at the moment $t=0$ ) and the static advancing contact angle (in the end of the spreading stage). Important to notice that according to Figure 4 the static receding contact angle, $\theta_{\mathrm{r}}$, does not remain constant during the second stage, but varies with time at concentrations below CWC.

Figure 5 shows the dependence of static advancing, $\theta_{a d}$, and static receding, $\theta_{r}$, contact angles on initial surfactant concentration, $C$, for aqueous solutions of surfactant SILWET L-77. Values of both contact angles presented in Figure 5 obtained from 
experimental data presented in Figure 4 (and similar data for other concentrations) in the following way: static advancing contact angle, $\theta_{\mathrm{ad}}$, is equal to the contact angle at the end of spreading process (initial stage in Figure 1); static receding contact angle is equal to the contact angle at the end of stage one, that is, at the moment when the radius of the droplet base starts to decrease. Note, the dependency of the radius of the droplet base on time always followed the dependency presented in the bottom part of Figure 1 independently of the surfactant concentration.

It looks like than not only advancing but also receding contact angles level off above CWC (for SILWET L-77 CWC=0.25 g/l [32]) Dependency of the static advancing contact angle on concentration presented in Figure 5 is in good agreement with the previous investigation [32].

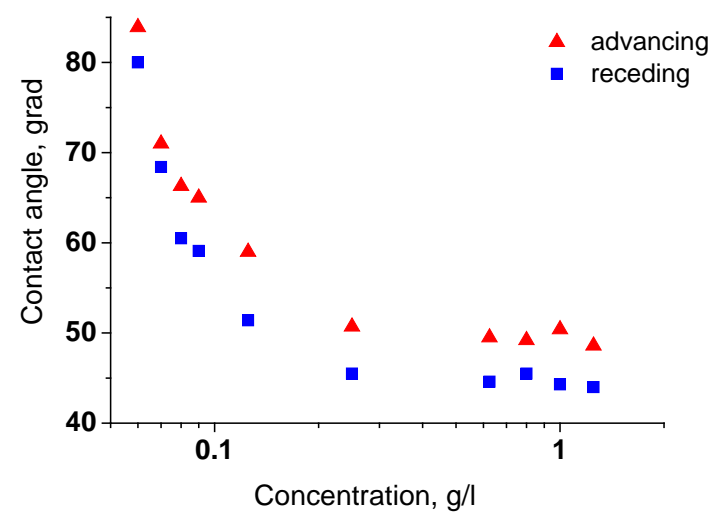

Figure 5. Dependence of advancing, $\theta_{a d}$, and receding, $\theta_{r}$, contact angles on initial surfactant concentration, $C$, for aqueous solutions of SILWET L-77.

Note that the statistic advancing contact angle was determined at the beginning of stage one, when the surfactant concentration was almost identical to that at the moment of 
deposition of the droplet. However, the situation is substantially different with the dependency of static receding contact angle on the surfactant concentration presented in Figure 5. The static receding contact angle was determined in the end of the first stage, when the surfactant concentration could be considerably higher as compared with the initial concentration because of evaporation. Note that in the case of concentrations below CWC the receding contact angle continued to decrease over the whole duration of the second stage of evaporation process. That is, the actual concentration is different from the initial one.

Comparison of the experimental data for evaporation of surfactant solutions with the theoretical predictions for pure liquids

In what follows the theoretical predictions for pure water are compared with the experimental results for aqueous surfactant solutions. Note again that both advancing and receding contact angles and their dependences on surfactant concentrations were extracted from experimental data. These angles presented in Figure 5 are very much different from those for water. According to the following results the kinetics of evaporation of surfactant solutions is very similar to that of pure aqueous droplets. The main differences in the case of surfactant solutions are (i) the lower values of initial contact angles and as a consequence (ii) larger initial radiuses of the droplet base at all concentrations; (iii) dependency of the receding contact angle on time at concentrations below CWC.

Figure 3 confirms that all slopes of $\mathrm{V}^{2 / 3}(\mathrm{t})$ linear dependences are equal to that of pure aqueous droplet within experimental error. According to Equation (7) these slops are proportional to the parameter $\beta$. Figure 3 and Equation (7) confirms that the parameter $\beta$ 
does not depend on the concentration of surfactants and, hence, the rate of evaporation does not according to Equation (2).

In the case of evaporation of trisiloxane solutions we used parameters $\beta$ calculated according to the procedure presented in [28] and shown in Figures S3 and S4. However, in the case of comparison presented in Figure 3 as well as comparison with experimental data on evaporation of SDS solutions presented in [20] a different procedure was used. That is below the parameter $\beta$ was calculated for cases mentioned above directly from the experimental data according to the following procedure. Integration of Equation (2) results in:

$V(t)-V_{0}=-\beta \int_{0}^{t} F[\theta(\hat{t})] L(\hat{t}) d \hat{t}$.

Let us denote $x(t)=\int_{0}^{t} F[\theta(\hat{t})] L(\hat{t}) d \hat{t}$, then Eq.(12) takes the following form:

$$
V(t)=-\beta x(t)+V_{0}
$$

The dependency of the volume of time $V(t)$ is known from the experiment; $x(t)$ is calculated using experimental values of $\theta(\mathrm{t})$ and $\mathrm{L}(\mathrm{t})$. Applying numerical integration over time (second order integration method), plotting V(t) vs. $\mathrm{x}(\mathrm{t})$ and fitting it with the linear dependence gives the required value of the parameter $\beta$ for each particular experimental run. Calculations according to the described procedure show that within the limits of experimental errors it can be concluded that $\beta$ does not depend on surfactant concentration for all the temperatures and relative humidities studied. The same is true for the SDS solutions studied by Doganci et al. [20]. The latter allowed applying the earlier developed theory for pure water for the case under consideration (Figures S3 and S4). 
It was found that the experimental data follow the predicted universal curve during the first stage of evaporation process for all investigated temperatures, relative humidities and concentrations (72 sets of $\theta, \mathrm{V}$ and $\mathrm{L}$ vs. $\mathrm{t}$ data).

However, the situation is more complex for the second stage of the spreading/evaporation, though the agreement with the theory predictions is still rather good. Figure 6 shows the example of data for one investigated conditions $(\mathrm{H}=30 \%$, $\mathrm{T}=30^{\circ}$ ) for concentrations below and above CAC; all other investigated cases shows the same behaviour.

It is seen from Figure 6 that there is a very good agreement with the theory predictions at concentrations above CAC and there are deviations from the theory predictions at concentrations below CAC. This may be understood considering that for the $0<\mathrm{C}<$ CAC concentration range the air/liquid and solid/liquid interfacial tensions change as the evaporation progress due to the increase of concentration. The receding contact angle decreases as concentration increases in the range $\mathrm{C}<\mathrm{CWC}$. The latter phenomenon was not included in either the computer simulations or the theory above. This may also explain why the agreement between theory and experiment for pure water is similar to that of the more concentrated surfactant solutions at concentrations above CWC.

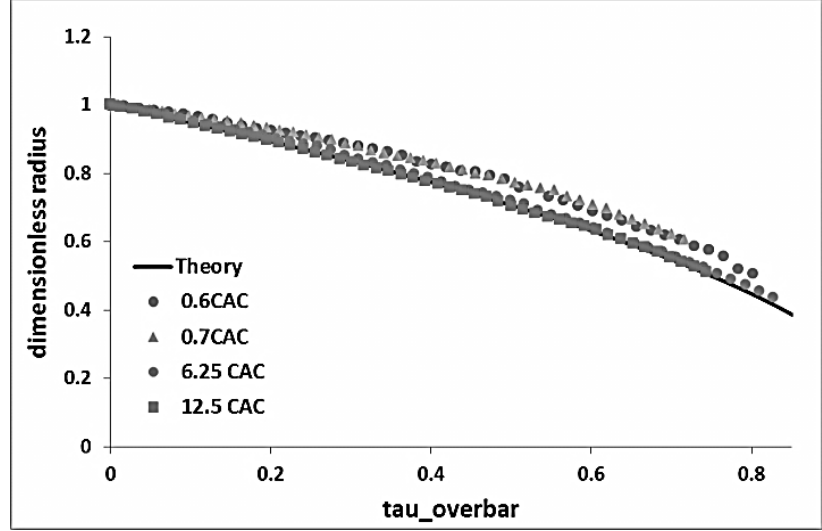


Figure 6. Comparison of the experimental results with the universal curve predicted by the theory for SILWET L77 solutions for the second stage of evaporation. Example for $\mathrm{H}=30 \%, \mathrm{~T}=30^{\circ}$.

Note once more, to plot the dependences presented in Figure 6 experimental values of advancing and receding contact angles were used.

In Figure 7 the experimental data published by Doganci et al. [20] for their experiments using SDS surfactant $\left(55 \% \mathrm{H}, 21^{\circ} \mathrm{C}\right)$ together with our results for SILWET L-77 $(90 \%$ $\mathrm{RH}, 18^{\circ} \mathrm{C}$ ) are presented. Figure 7 proves that the agreement with theory predictions is similar for both surfactants although the scattering around the universal curve for the second evaporation stage seems to be higher for the SDS data.

Note, solid substrates used for the SILWET L77 and for the SDS solutions were different and it was the reason why we used a different procedure (presented above) for calculation of the parameter $\beta$ in the case of SDS solutions.

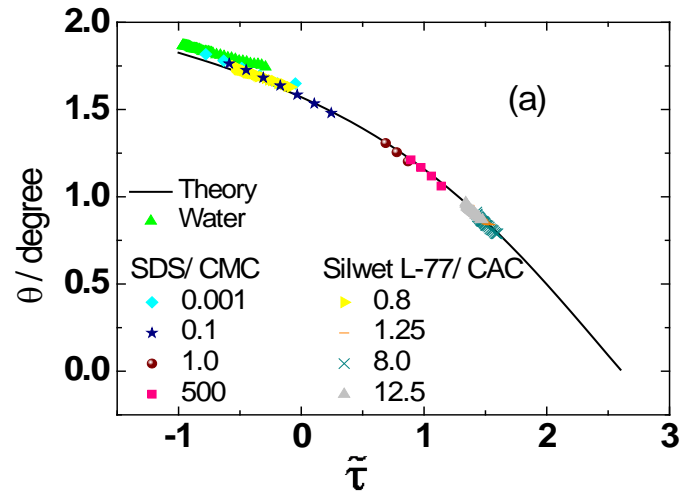




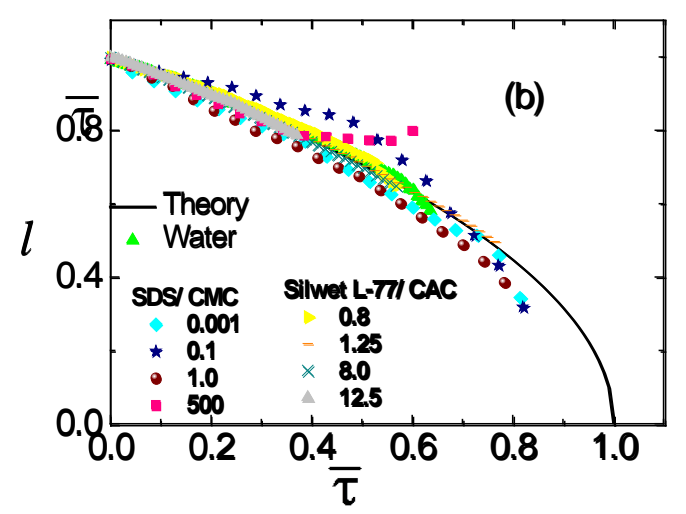

Figure 7. Comparison of the universal behaviour predicted by the theory and experimental results of SDS [20] and of SILWET L77 solutions for (a) first stage of evaporation and (b) second stage of evaporation.

A qualitative explanation of deviation from the theory predictions at low concentration of surfactants

There are two different processes causing the change in the surfactant bulk concentration during spreading/evaporation: (i) the concentration decreases due to depletion caused by the adsorption and (ii) increases because of decrease of volume due to evaporation. The depletion of volume concentration due to adsorption of SILWET L77 on both liquid/air and liquid/solid can be important at small size of droplets used in our experiments. It becomes more important at the decrease of the droplet size and surfactant concentration. According to [25] the trisiloxane surfactant with 8 oxyethylated groups, having properties very close to SILWET L77, had adsorption at solution air interface very close to saturation at bulk concentrations above 0.01 CAC. At concentrations above 0.1 CAC used in our experiments adsorption is about $6 \mathrm{E}-4 \mathrm{~g} / \mathrm{m}^{2}$. Adsorption on the Teflon/solution interface according to our preliminary study is about $5 \mathrm{E}-3 \mathrm{~g} / \mathrm{m}^{2}$ for the bulk concentration equal to CAC and about $7 \mathrm{e}-4 \mathrm{~g} / \mathrm{m}^{2}$ for 
concentration 0.1 CAC. It is easy to estimate that for the hemispherical droplet with volume used in this study (about $3.5 \mathrm{~mm}^{3}$ ), about $10 \%$ of initial amount of surfactant will be adsorbed at the bulk concentration equal to CAC and about $35 \%$ will be adsorbed at the initial bulk concentration of 0.1 CAC. The latter means that the adsorption will result in a substantial decrease of the bulk concentration inside the droplet and as a result in a significant change of the receding contact angle.

\section{The third stage of evaporation}

The third stage of spreading evaporation is much shorter than the first and second stage of evaporation (see Figure S6 in Supporting Information for experimental data). The initial spreading stage is too short and is not shown in Figure S6. The contact angle deviates from the static receding contact angle, $\theta_{\mathrm{r}}$, and decreases relatively fast during the third stage. The latter is the problem for a theoretical description of the process during this stage. It is well-known that the receding contact angle can decrease at relatively high receding velocity, that is, if the capillary number, $C a=\frac{U \mu}{\gamma}$, Ca $10^{-4}-10^{-}$

${ }^{3}$. However, a very simple estimation of a capillary number during the third stage show that during this stage $C a \sim 10^{-7}-10^{-8}$, which is much smaller than $10^{-4}$. This means that the considerable deviation of the receding contact angle, which takes place during the third stage has nothing to do with Ca number.

However, during this stage a sharp transition from $\theta_{\mathrm{r}}>90^{0}$ (non-wetting) to $\theta_{\mathrm{r}}<90^{0}$ (partial wetting) takes place (see Figure S7 in Supporting Information). It is well established that both static advancing and static receding contact angles on smooth homogeneous substrates (like we used in our experiments) are completely determined by surface forces action in a vicinity of the three phase contact line [33, 34]. The latter forces are well known in the case of partial wetting (modified DLVO theory), but very 
little is known in the case of non-wetting. It is possible to assume that in the case of non-wetting those forces are considerably different from the case of partial wetting and that just this transition is responsible for the occurrence of the third stage.

\section{Conclusions}

Kinetics of a simultaneous spreading/evaporation process of droplets of aqueous solutions of the super-spreader SILWET L77 has been investigated. Increasing the surfactant concentration reduces the static advancing contact angle from $118 \pm 2^{\circ}$ for a pure aqueous droplet down to about $50^{\circ}$ as expected. Four different stages of process were found: a) Spreading stage. An initial stage, which corresponds to the spreading stage already described by Ivanova et al. [26, 32], this stage ends when the contact angle reaches the value of static advancing contact angle, $\theta_{a d}$. The characteristic times for this stage found at all concentrations, temperatures and relative humidities investigated are in agreement with those reported in Refs. [26, 32] for trisiloxane solutions. b) The first stage takes place at constant droplet perimeter and decreasing contact angle. This stage ends when the contact angle reached the value of static receding contact angle, $\theta_{r}$. During this stage a very good agreement has been observed between our experimental data (time dependence of $\theta$ for the pinned droplet) with the universal behaviour predicted by the theory of Semenov et al. [28]. The data of Doganci et al. [20] for SDS solutions also show a very good agreement with the theoretical predictions. c) The second stage takes place at constant $\theta=\theta_{r}$ while the droplet perimeter decreases. In this case the theory also predicts a universal curve for the time dependence of the droplet perimeter. The agreement of the experiments with the theory is also very good at concentrations above CWC/CMC in the case of SILWET L77/SDS solutions. However, during the second stage static receding contact angle does not remain constant at 
concentrations below CWC/CMC and there are some deviations from the predicted universal behaviour. At such low concentrations the surface tensions show strong dependence on bulk surfactant concentration. During all three mentioned stages of spreading/evaporation the volume to power $2 / 3, \mathrm{~V}^{2 / 3}$, shows a linear dependence on time. d) The third stage is characterized by a simultaneous decrease of the perimeter and the contact angle till the complete disappearance of the droplet. So far there is no theoretical description for this stage, which for droplet radius small enough should include the contributions of the DLVO and non-DLVO forces.

\section{Acknowledgments}

This work was supported in part by MICINN under grant FIS2012-28231-C02-01, by ESA under grants FASES and PASTA, COST MP1106 project, and by Engineering and Physical Sciences Research Council, UK, grant EP/D077869/1.

\section{Supporting Information}

The geometry of evaporation of a spherical cap droplet (Figure S1); Sketch of the solid support adopted for calculations of parameter $\beta$ (Figure S2); Calculated parameter $\beta$ as a function of temperature and humidity (Figures S3, S4); Diagram of the experimental devise used (Figure S5); Illustrations for third stages of evaporation (Figures S6, S7). This material is available free of charge via the Internet at http://pubs.acs.org/4authors. 


\section{References}

1. Semenov, S.; Starov, V.M.; Velarde, M.G.; Rubio, R.G. Droplets Evaporation: Problems and Solutions. Eur. Phys. J. Special Topics 2011, 197, 265-278.

2. Bourges-Monnier, C.; Shanahan, M. Influence of Evaporation on Contact Angle. Langmuir 2005, 11, 2820-2829.

3. Sefiane, K.; Tadrist, L.; Douglas, M. Experimental study of evaporating waterethanol mixture sessile drop: influence of concentration. Int. J. Heat Mass Tran. 2003, 46, 4527-4534.

4. Pautsh, A.G.; Shedd, T.A. Spray impingement cooling with single- and multiple-nozzle arrays. Part I: Heat transfer data using FC-72. Int. J. Heat Mass Transfer, 2005, 48, 3167-3175.

5. Shedd, T.A.; Pautsh, A.G. Spray impingement cooling with single- and multiple-nozzle arrays. Part II: Visualization and empirical models. Int. J. Heat Mass Transfer, 2005, 48, 3176-3184.

6. Bhardwaj, R.; Longtin, J.P.; Attinger, D. Interfacial temperature measurements, high-speed visualization and finite-element simulations of droplet impact and evaporation on a solid surface. Int. J. Heat Mass Transfer, 2010, 53, 3733-3744

7. Burger, M; Schmehl, R.; Prommersberger, K.; Schafer, O.; Koch, R.; Wittig, S. Droplet evaporation modelling by the distillation curve model: accounting for kerosene fuel and elevated pressures. Int. J. Heat Mass Transfer, 2003, 46, 4403-4412.

8. Johnson, M.V.; Zhu, G.S.; Aggarwal, S.K.; Goldsborough, S.S. Droplet evaporation characteristics due to wet compression under RCM conditions. Int. J. Heat Mass Transfer, 2010, 53, 1100-1111. 
9. Kristyadi, T.; Depredurand, V.; Castanet, G.; Lemoine, F.; Sazhin, S.S.; Elwardany, A.; Sazhina, E.M.; Heikal, M.R. Monodisperse monocomponent fuel droplet heating and evaporation. Fuel, 2010, 89, 3995-4001.

10. Zoby, M.R.G.; Navarro-Martinez, S.; Kronenburg, A.; Marquis, A.J. Evaporation rates of droplet arrays in turbulent reacting flows. Proc. Combust. Inst. 2011, 33, 2117-2125.

11. Miliauskas, G.; Sinkunas, S.; Miliauskas, G. Evaporation and condensing augmentation of water droplets in flue gas. Int. J. Heat Mass Transfer, 2010, 53, 1220-1230.

12. Boulet, P.; Tissot, J.; Fournaison, L. Enhancement of heat exchangers on a condenser using an air flow containing water droplets. Appl. Thermal Eng. 2013, $50,1164-1173$

13. Grant, G; Brenton, J.; Drysdale, D. Fire suppression by water sprays. Prog. Energy Combust. Sci. 2000, 26, 79-130.

14. Gupta, M.; Pasi, A.; Ray, A.; Kale, S.R. An experimental study of the effects of water mist characteristics on pool fire suppresion, Exp. Thermal Fluid Sci. 2013, 44, 768-778.

15. Turner, M.R.; Sazhin, S.S; Healey J.J.; Crua, C.; Martynov, S.B. A breakup model for transient Diesel fuel sprays. Fuel, 2012, 97, 288-305.

16. Chen, J.; Liao, W.S.; Chen, X.; Yang, T.; Wark, S.E.; Son, D.H.; Batteas, J.D.; Cremer, P.S. Evaporation-induced assembly of quantum dots into nanorings. ACS Nano 2009, 3(1), 173-180

17. Talbot, E.L.; Berson, A.; Brown, P.S.; Bain, C.D. Evaporation of picoliter droplets on surfaces with a range of wettabilities and thermal conductivities. Phys. Rev. E, 2012, 85(6), 061604 
18. Hu, H.; Larson, R. G. Evaporation of a sessile droplet on a substrate. J. Phys. Chem. B. 2002, 106 (6), 1334.

19. Cioulachtjian, S.; Launay, S.; Boddaert, S.; Lallemand, M. Experimental investigation of water drop evaporation under moist air or saturated vapour conditions. Int. J. Therm. Sci. 2010, 49, 859-866.

20. Doganci, M.D.; Sesli, B.U.; Erbil, H.Y. Diffusion-controlled evaporation of sodium dodecyl sulfate solutions drops placed on a hydrophobic substrate. $J$. Colloid Interf. Sci. 2011, 362, 524-531.

21. Sultan, E.; Boudaoud, A.; Amar, M.B. Evaporation of a Thin Film: Diffusion of the Vapour and Marangoni Instabilities. J. Fluid Mech. 2005, 543, 183-202.

22. Rednikov, A.Ye.; Colinet, P. Truncated versus Extended Microfilms at a VaporLiquid Contact Line on a Heated Substrate. Langmuir 2011, 27, 1758-1769.

23. Ajaev, V.S. Spreading of Thin Volatile Liquid Droplets on Uniformly Heated Surfaces. J. Fluid Mech. 2005, 528 279-296.

24. Moroi, Y.; Rusdi, M.; Kubo, I. Difference in surface properties between insoluble monolayer and adsorbed film from kinetics of water evaporation and BAM image. J. Phys. Chem. B 2004, 108, 6351-6358

25. Ritacco, H.; Ortega, F.; Rubio, R.G.; Ivanova, N.; Starov, V.M. Equilibrium and dynamic surface properties of trisiloxane aqueous solutions. Part 1. Experimental. Colloids and Surfaces A 2010, 365, 199-203.

26. Ivanova, N.; Starov, V.; Johnson, D.; Hilal, N.; Rubio, R.G. Spreading of aqueous solutions of trisiloxanes and conventional surfactants over PTFE AF coated silicon wafers. Langmuir 2009, 25, 3564-3570. 
27. Semenov, S.; Starov, V.M.; Rubio, R.G.; Agogo, H.; Velarde, M.G. Evaporation of sessile water droplets: Universal behaviour in presence of contact angle hysteresis. Colloids and Surfaces A 2011, 391, 135 - 144

28. Semenov, S.; Starov, V.M.; Rubio, R.G.; Velarde, M.G. Instantaneous distribution of fluxes in the course of evaporation of sessile liquid droplets: Computer simulations. Colloids and Surfaces A 2010, 372, 127 - 134.

29. Semenov, S., Starov, V., Rubio, R., Velarde, M. Computer simulations of evaporation of pinned sessile droplets: Influence of kinetic effects. Langmuir 2012, 28, 15203-15211.

30. Semenov, S., Starov, V., Rubio, R. Evaporation of pinned sessile microdroplets on highly-heat conductive substrates. Computer simulations. Eur. Phys. J. Special Topics 2013, 219, 143-154.

31. Svitova, T.; Hill R.M.; Radke, C.J. Adsorption layer structure and spreading behaviour of aqueous non-ionic surfactants on graphite. Colloids and Surfaces A 2001, 183-185, 607-620

32. Ivanova, N.; Starov, V.M.; Rubio, R.G.; Ritacco, H.; Hilal, N.; Johnson, D. Critical wetting concentrations of trisiloxane surfactants. Colloids and Surfaces A. 2010, 354, 143-148.

33. Starov, V., Velarde, M., Radke, C. Wetting and Spreading Dynamics. Surfactant Science Series. 138. Taylor \& Frances, 2008, pp 515.

34. Starov, V. Static contact angle hysteresis on smooth, homogeneous solid substrates. Colloid Polym. Sci. 2013, 291, 261-270.

35. Sefiane, K. The coupling between evaporation and adsorbed surfactant accumulation and its effect on the wetting and spreading behaviour of volatile drops on a hot surface. J. Petrol. Sci. Eng. 2006, 51, 238-252. 
36. Soboleva, O.A.; Summ, B.D. The kinetics of dewetting of hydrophobic surfaces during the evaporation of surfactant solution drops. Colloid J. 2003, 65, 89-93.

37. Gutiérrez, G.; Benito, J.M.; Coca, J.; Pazos, C. Vacuum evaporation of surfactant solutions and oil-in-water emulsions. Chem. Eng. J. 2010, 162, 201207.

38. Gokhale, S.J.; Plawsky, J.L.; Wayner, P.C. Spreading, evaporation, and contact line dynamics of surfactant-laden microdrops. Langmuir 2005, 21, 8188-8197

39. Alexandridis, P.; Munshi, S.Z.; Gu, Z. Evaporation of water from structured surfactant solutions. Ind. Eng. Chem. Res. 2011, 50, 580-589.

40. Picket, R.G.; Bexon, R. Evaporation of sessile or pendant drops in still air. J. Colloid Interf. Sci. 1977, 61, 336-350.

41. Wagner, R.; Richter, L.; Weißmuller, J.; Reiners, J.; Klein, K.D.; Schaefer, D.; Stadtmuller, S. Silicon-modified carbohydrate surfactants: IV. The impact of substructures on the wetting behavior of siloxanil-modified carbohydrate surfactants on low-energy surfaces. Appl. Organomet. Chem. 1997, 11, 617-632. 
TOC graphic

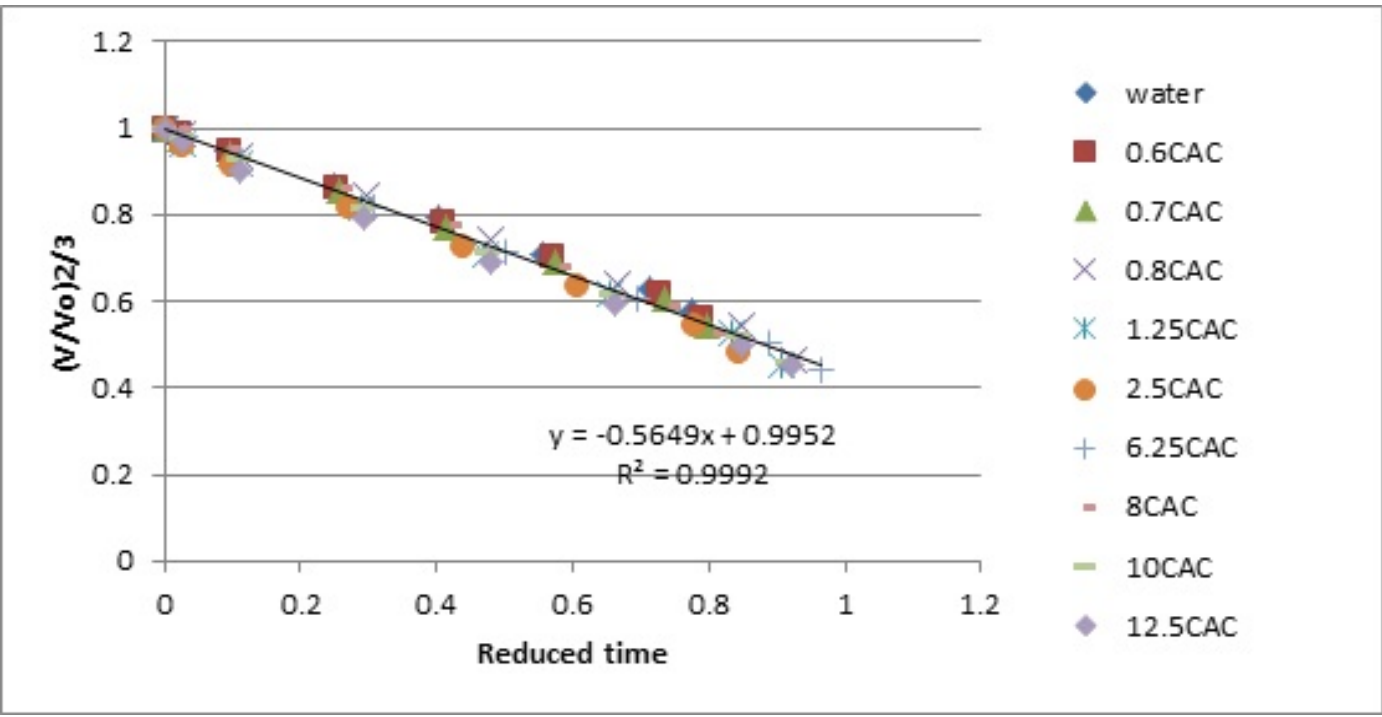

